Z Gerontol Geriat 2012 · 45:5-5 DOI 10.1007/s00391-011-0259-1

Online publiziert: 20. Januar 2012

(c) Springer-Verlag 2011

\title{
H. Förstl
}

Klinik für Psychiatrie und Psychotherapie, Technische Universität München

\section{Demenz}

\section{Weshalb es sich lohnt, dem Thema noch einige Jahrzehnte Aufmerksamkeit zu schenken}

Es gibt eine sehr einfache Grundregel in der Natur: Wenn etwas bereits sehr lange gedauert hat, wird es voraussichtlich auch noch lange andauern. Dies bedeutet keineswegs, dass alles auf Dauer so bleiben muss, wie es ist, und es ist schon gar keine Rechtfertigung die Hände schicksalsergeben in den Schoß zu legen. Die Grundlagenwissenschaften haben schon einige mögliche Ansätze zur Lösung des Demenzproblems geliefert. Die Industrie arbeitet daran, diese Ideen an die $\mathrm{Pa}$ tienten zu bringen, und dies funktioniert teilweise schon recht gut - was die Biologie anbetrifft. Die Nebenwirkungen sind beachtlich, und die Wirkungen sind von wissenschaftlichem Interesse, allerdings noch nicht von Nutzen für die dementen Patienten von heute, vielleicht auch nicht für die dementen Patienten der Zukunft. Aber verhindern könnte man die Demenz - und dabei meine ich eigentlich immer die kognitiven Defizite im Zusammenhang mit den typischen Alzheimer-Hirnveränderungen - vielleicht eines Tages. Dies wäre eine durchaus hoffnungsvolle Perspektive für die Enkelgeneration.

Für viele Jahrzehnte wird die Aufgabe Alzheimer etc. noch viele Menschen professionell und privat beschäftigen eigentlich uns alle. Bei den Demenzen und den Erkrankungen, die dazu beitragen, handelt es sich um ein gesellschaftliches Problem, also ein Problem von uns allen, von jedem Einzelnen. Mehrere Beiträge in diesem Schwerpunktheft nehmen darauf Bezug. Solange unklar ist, wie kognitive Reserven am geschicktesten und zuverlässigsten aufzubauen sind, bleibt die medizinische Prävention gewöhnli- cher, beeinflussbarer Risikoerkrankungen von der Depression zum Diabetes und Bluthochdruck Bürgerpflicht. Der schlanke und agile Nichtraucher meint vielleicht, die prävalenten und signifikanten Probleme Bewegungsmangel, Fettleibigkeit und Nikotinismus seien besonders leicht in den Griff zu bekommen. Da man um die Nachteile dieser Merkmale schon lange weiß und dennoch kein sportlicher Ruck durch Deutschland und die Welt geht, scheint belegt, dass Vernunft, lebenslanger Lebensstil und der Verstand im Alter auf recht komplizierte Art verflochten sind. Auch im hohen Alter und noch im Stadium der symptomatischen Alzheimer-Krankheit bleibt der gesamte Lebensstil von der Diät bis zur Bewegung sicherlich von hoher Bedeutung, man wüsste nur genau mehr darüber. Allerdings beschränkt sich die medizinische Betrachtung der Demenzen häufig auf Themen der modernen Diagnostik und pharmakologische Interventionen. Diesen Fehler macht dieses Schwerpunktheft nicht.

Hans Förstl

\section{Korrespondenzadresse}

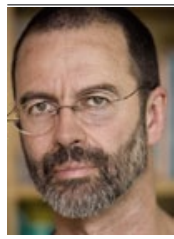

Prof. Dr. H. Förstl

Klinik für Psychiatrie und Psychotherapie Technische Universität München Ismaninger Str. 22 81675 München hans.foerstl@ Irz.tu-muenchen.de 